\title{
Experimental Study of Crossing Angle Collision ${ }^{\dagger}$ \\ T. chen
}

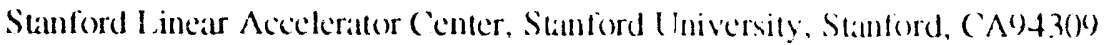

1). Ricc, 1). Rubin, D). Sagan, and M. Tigner

Newman I ahoratory, ('omell Iniversity, Ithaka, NY' 148.5 .3

\begin{abstract}
The non-linear coupling due to the beam-beam interaction with crossing angle has been studied. The major effect of a small $(-12 \mathrm{mrad})$ crossing angle is 10 excite $5 Q_{\mathrm{x}} \pm Q_{s}=$ integer coupling resonanec family on large amplitude partickes, which results in bad lifetime. (In the ( $\mathrm{YSSR}$, a small crossing angle ( 2.4mr) was crealed alt the IP' and a reasonate beam-beam lune-shift was achieved. The decaly ralle of the beam is measured as a function of horizontal lune with and without crossing angle. The theoretical analysis, simulation and experimental measurements hate a good agreement. The resonance strenght as a function of crossing angle is also meatsured.
\end{abstract}

\section{INTR()I)IICTI()N}

A primary objective of mexlem $e^{+} e^{-}$collider development is to achieve very high luminosity to meet the requirements of high energy physics. The design luminosity of these colliders, so called B-lactorics, D-Factories, etc., is about 50) times as high as that achieved in current colliders. To ohtain this luminosity, the new designs employ two rings, with each ring being filled with large number of bunches 10 make the collision rate at the single interation point large. The natural way to bring the two beams into collision and separating them thereafter is to have a small crossing angle. However. studies 11$]$ show that synchrohetatron resonances are excited by crossing angle beam-beam interaction. This paper analyges this problem, and provides the results of an experimemtal measurement. The conclusion of analysis, simulation, and experiment agrees reasonably well.

\section{ANAI.YTIC AI. ANAI.YSIS ANI) SIMIII.ATION}

The reason why synchrobetatron coupling is introduced by a crosesing angle is that. dese to the angle, lhe distance between a particle and the eenter of the counter bunch is modulated by the particle's longitudinal position. As a result, the beam-beam kick, which is a function of the distance, is modulated by the longitudinal motion too).

A resonance analysis medhed can be developed balsed on difference equations $[2]$. (onsidering horizontal and longitudinal planes, particle motion in a lincar ring with a thin. nomlinear kick can be described by

$x_{t+1}-2 \cos \mu_{x} x_{t}+x_{t-1}=-\beta_{x} \sin _{x} l\left(x_{t}+\tan d \cdot s_{t}\right) \cos s^{2}()$

$s_{t+1}-2 c(s) \mu_{s} s_{t}+s_{t-1} \approx 0$.

Where $\mathrm{I}(r)$ is the heam-beam kick, $\Phi$ is the crossing angle, and 1 stands for turn number. for small crossing angle, the longitudinal component of the kick is neglected. It is casy to sce that the linear solutions of ( 1$)$ are:

$x_{t}=A_{x} \cos \left(\mu_{x} t\right), \quad s_{t}=A_{s} \cos \left(\mu_{s} t\right)$.

As the first step approximation, insert (2) into the right-

+ Work supported in part by (Ine Department of lencrgy

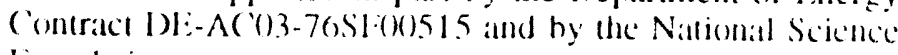
foundation. hand side of (1), and do a lourier expansion. Then, the righthand-side of tirst eyualton of (1) is written as:

$\frac{1}{2} \sum_{m, n}\left(m, n \cos \left|\left(m \mu_{x}+n \mu_{s}\right) t\right|+d_{m, n} \cos \left|\left(m \mu_{x}-n \mu_{s}\right) t\right|\right.$

Nalurally, a similar form of solution of (1) is expected:

$x_{t}=\frac{1}{2} \sum_{m, n} a_{m, n} \cos \left|\left(m \mu_{x}+n \mu_{s}\right) t\right|+b_{m, n} \cos \left|\left(m \mu_{x}-n \mu_{s}\right) t\right|$

Substitute (3), (4) into (1), the relation between driving terms and respense tems is found:

$(a, b)_{m, n}=\frac{(c, d) m_{1} n}{2 \sin \frac{1}{2}\left|(m+1) \mu_{x} \pm n \mu_{s}\right| \sin \frac{1}{2}\left|(m-1) \mu_{x} \pm n \mu_{s}\right|}$

Near resomances $(m+1) Q_{\mathrm{X}} \pm n()_{s}=$ integer, the denominator is small. Then, $(a, b)_{m, n}$ hats strong response lo $(c, d)_{m, n}$. Therefore, we can saly that $c m, n$ and $d_{m, n}$ drive these resomances.

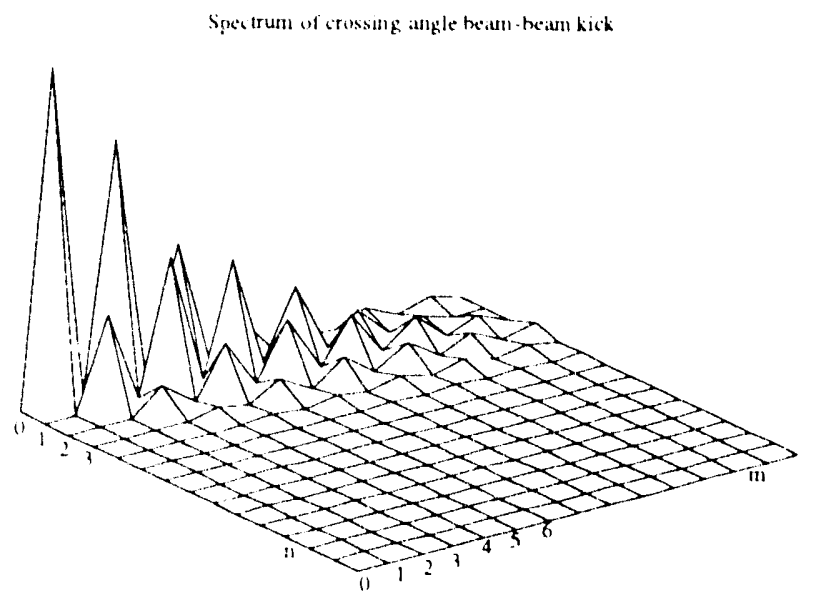

Figure (1). The power spectrum of the crossing angle beambeism kick

figure (1) shows the lwo-dimensional lipl power spectrum of the beam-beam kick. Irom the picture, one can casily see that the strongest driving terms are at $m=4, n=1$ and $m=6, n=1$. According (o) the previous analysis, hoth these (wo) terms will drive 5()$_{\mathrm{X}}+\mathrm{Q}_{S}=$ integer resonances. It is natural to conclude that the $5 Q_{x} \pm Q_{s}=$ integer resonances are the strongest coupling resonances.

In order 10 analysec the effect of a crossing angle, a compuler simulation similar to Piwinski's work|3| was made. The storage ring was moklel by a lincar ring and a thin-kick beam-beam interaction with crossing angle. Three dimensional motion is simulated. Particles are lannched in 6 dimensional phase space with $6 \sigma$ amplitudes. The program scans the horizontal fractional tunc from () $10 \mathrm{l}$. The maximum amplitude of all particles ever reached during the $I(x)()$-tum tracking is recorded ats a function of horizontal tune. ligure (2) plots the maximum horizontal amplitude versus tractional tunc. It shows that. besides the one-dimensional 
fractional tune. It shows that, besides the one-dimensional resonances which exist also in head-on collision, the strongest coupling resonances are $5 \mathrm{Q}_{\mathrm{X}} \pm\left(\mathrm{Q}_{s}=\right.$ integer family.

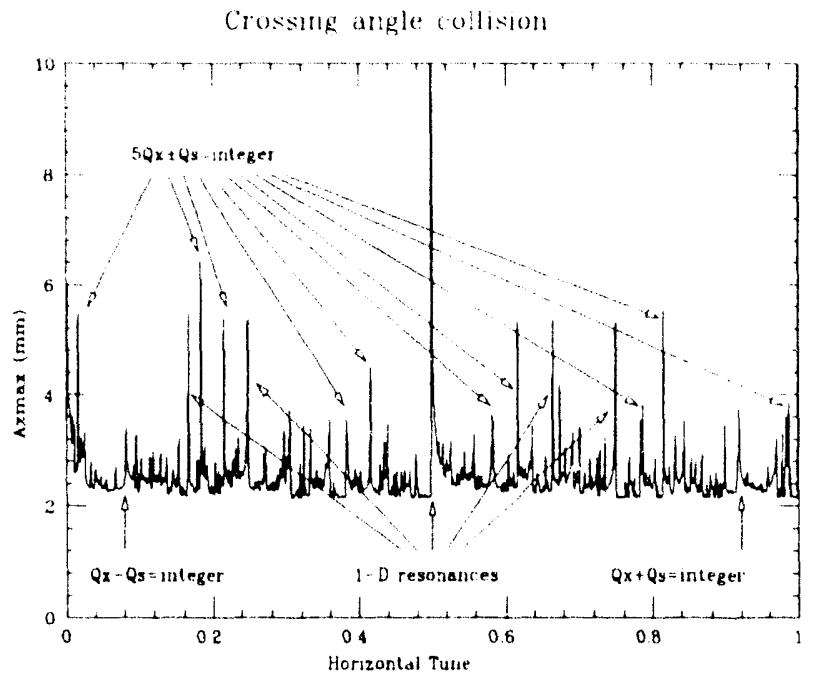

Figure (2) Maximum horizontal amplitude vs. tune for crossing angle collision. $\left(Q_{\mathrm{S}}=0.081\right)$

\section{EXPERIMENTAL MEASUREMIENT}

The experiment is designed to observe the $5 Q_{x}+Q_{s}$ resonance excited by the crossing angle collision, which is predicted by the theory in previous section. The experiment is based on the setup of the CESR crossing angle experiment ${ }^{[4]}$. CESR has been running with multi-bunch mode ( 7 bunches of $e^{-}$on 7 bunches of $e^{+}$). The key point of making multi-bunch mode possible is to separate bunches at crossing points around the ring except at the interaction point where the detector is located. In CESR, four electrostatic separators are used to separate electron and positron orbits at parasitic crossing points. As shown in figure (3), the orbits (thin lines) are separated at 13 would be collision points, but merged between the two south (lower) separators, including the IP where the collision takes place. The crossing angle lattice is essentially a modified version of the normal operation lattice with the bunches separated at the collision points except the IP. An anti-symmetric voltage applied to the solth separators will create anti-symmetric orbits about the IP. This is displayed in figure (3) as the thick lines.

The experiment was performed in a way similar to the simulation. The strong-weak bean-beam interaction is achieved by colliding a $2 \mathrm{~mA}$ beam on $10 \mathrm{~mA}$ bean. The beam size and beam current decay rate is measured while scanning the horizontal tune in the $5 Q_{x}+Q_{s}$ resonance region. $A$ high decay rate peak was observed on the resonance when the crossing angle was turned on. However, the peak disappeared when the crossing angle was turned off. Iigure (4) shows the tune scan data with and without crossing angle. For comparison, the simulation results are shown in figure (4) too. One can easily see the agreement between them. Note that the vertical axis represents different quantities in experimental data and simulation. The reason is that the calculation is only qualitative. Nevertheless, they both reflect the same physical phenomena. Meanwhile, the vertical beam size is measured. No beam blow up is observed at the same resonance, with or without crossing angle. This implies that this effect applies only on beam tail, which is what the theoretical analysis and simulation predicted.

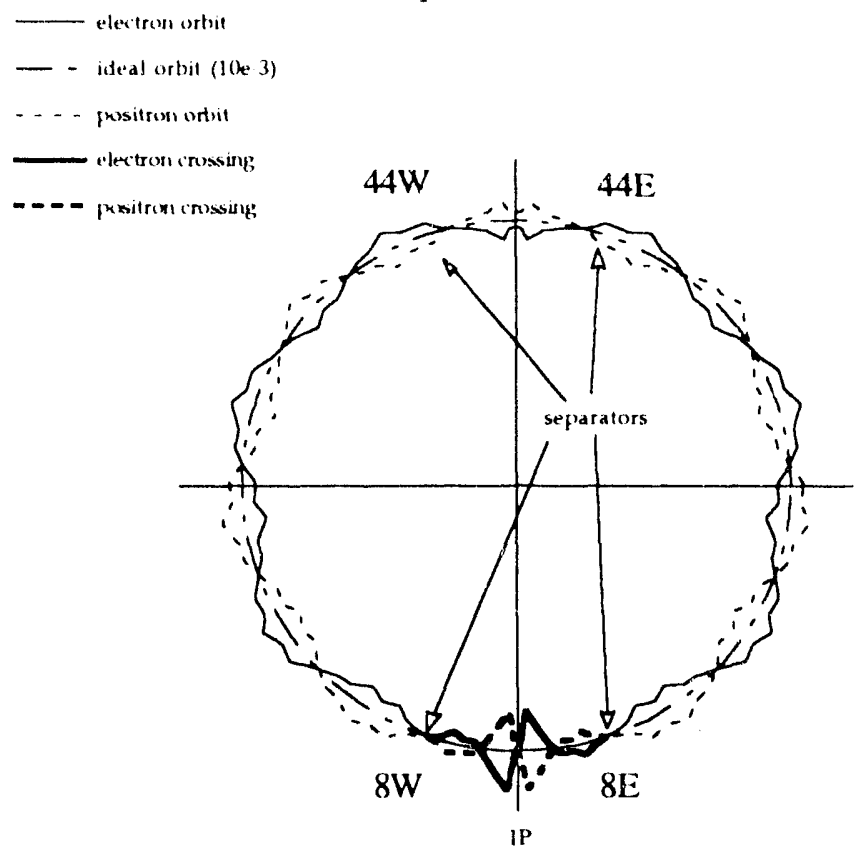

Figure(3). Diagram of the orbits for crossing angle experiment

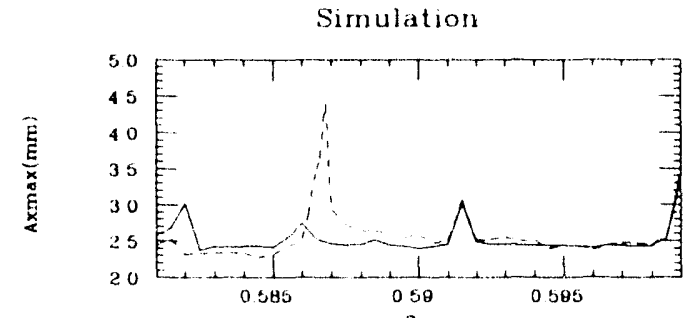

Experimental data

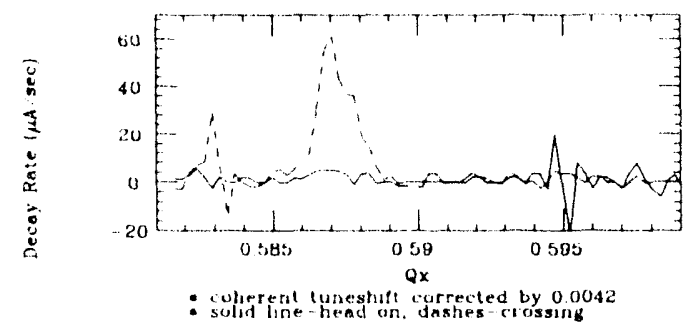

Figure (4). (a)upper: Simulation result, maximum amplitude versus horizontal tune. (b)lower: Experimental data, decay rate as a function of horizontal tune.

A iwo dimensional tune scan was also performed to check the resonance. The result with crossing angle on is shown in ligure (5). In this part of experiment, strong-strong beambeam interaction was employed, because, a weak beam cannot survive after crossing the resonance many times. The $5 Q_{x}+Q_{s}$ resonance corresponds to the light vertical line on the left. With this result, the resonance is better identified due to its consistent appearance and independent of vertical tune.

The resonance strength, in terms of peak decay rate, is 


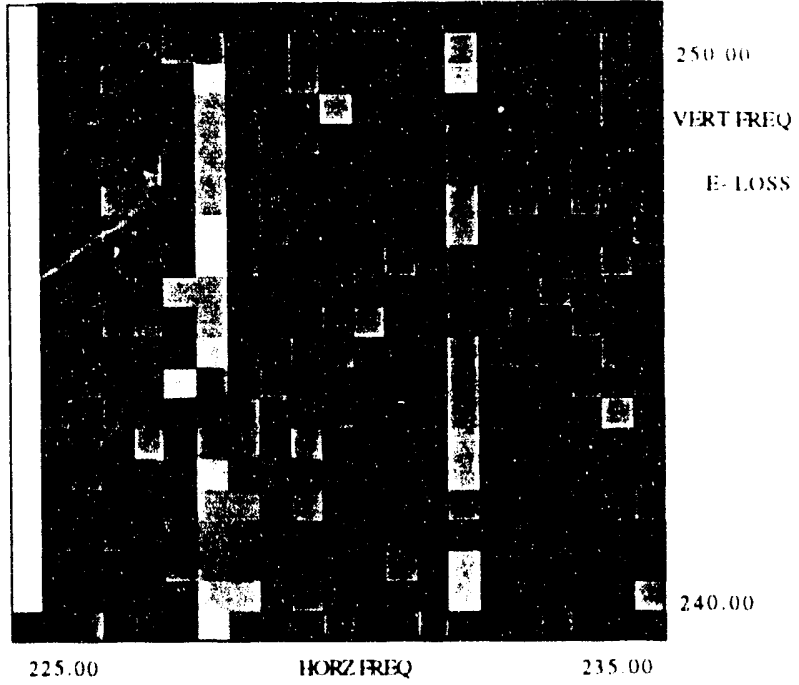

Figure (5) Electron decay rate measured in two dimensional tune scan with 2 mrad crossing angle. The lighter shade indicates higher decay rate. The horizontal and vertical tune frequencies, the product of the tune and the revolution frequency, are in $\mathrm{kHz}$. (The revolution frequency is $390 \mathrm{kHz}$ ). also measured as a function of crossing angle. Figure (6) plots the measured result. Each line in the picture is from a single tune scan with certain crossing angle. The crossing angle ranges from about $\pm 1.4 \mathrm{mrad}$ to $\pm 2.5 \mathrm{mi}$ id. For crossing angle smaller than $\pm 1.4 \mathrm{mrad}$, there is no clear decay rate peak being measured.

Crossing Angle Experiment

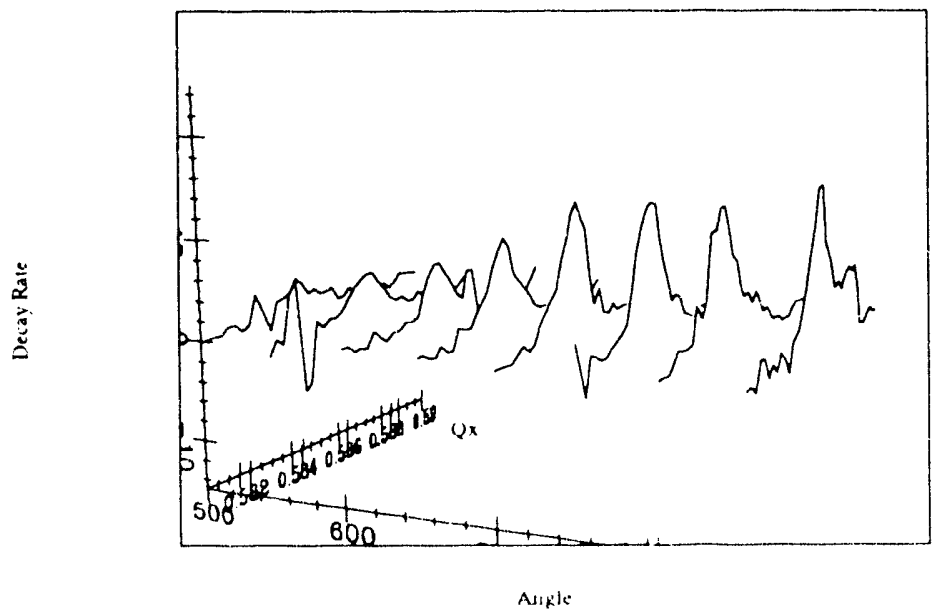

Figure (6). Tune scans versus crossing angle.

The maximum decaly rate from figure $(6)$ is plotted as a function of the half crossing angle in figure (7), onc can easily see the rise of the resonance strength as the crossing angle increases. The simulation result is also ploted for comparison. Again, the quantities in vertical axis are different, so that the comparison is only qualitative However, from both plots, a saturation effect can be seen Simulation shows that the saturation goes up $10 \pm 12$ mrad
Unfortunately, the crossing angle in the experiment cannot go larger, because it is limiled by machine aperture. The last dalla point raises again. The reason may be that the crossing angle has been pushed to the limit of the physical aperture at this angle. The light physical aperture certainly enhances the decay rate. We also cannot exclude other driving sources.

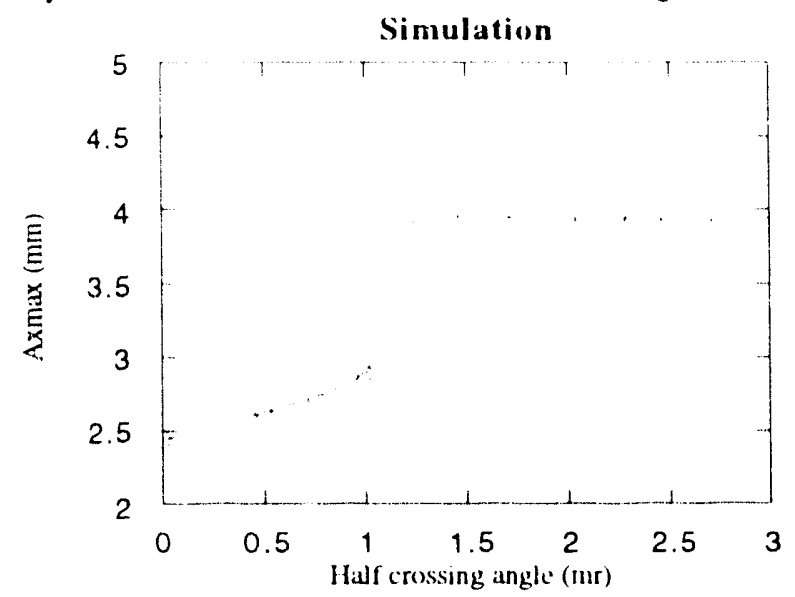

(a) Maximum amplitude on the resonance vs crossing angle.

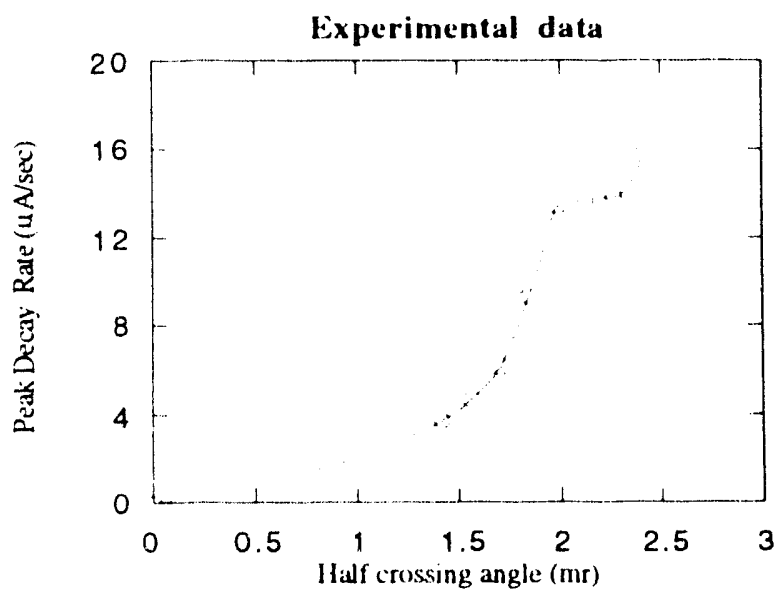

(b). Pu ik decay rate on the resonance vs. crossing angle Figuie (7). Resonance strength as a function of crossing angle.

\section{CONCLUUSIONS}

The study shows a good consistency arnong analytical analysis, eomputer simulation and experiment on the strongest coupling resonance family excited by the crossing angle beam-bean interaction. This resonance family. $5\left(Q_{X} \pm Q_{S}=\right.$ integer, will result in a bad lifetime in operation.

\section{ACKN()WI.EDCiMENI:}

Authors would like to sincerely thank (XSR accelerator physicists and operation group for their help for the experiment.

\section{RIITERIENCTS}

11) A. Piwinski, ILiEl 'Trans. NS-24, p1408 (1977).

12] Peggs, S. and Talman, R., "Nonlincar Problems in Accelerator Physics," Ann. Rev. Nucl. Part. Sci. , vol. $36,287,1986$.

13) Piwinski, A., "Simulations of Crab Crossing in Storage Rings," SI A (-PIB-5430, Ieb. 1991.

(4) Rubin, 1), and ei al, "Beam-beam Interaction with a Ilorizonlal crossing Angle," to be published in Nuclear Instrumbents and Methods A or (INS 92/1183. 

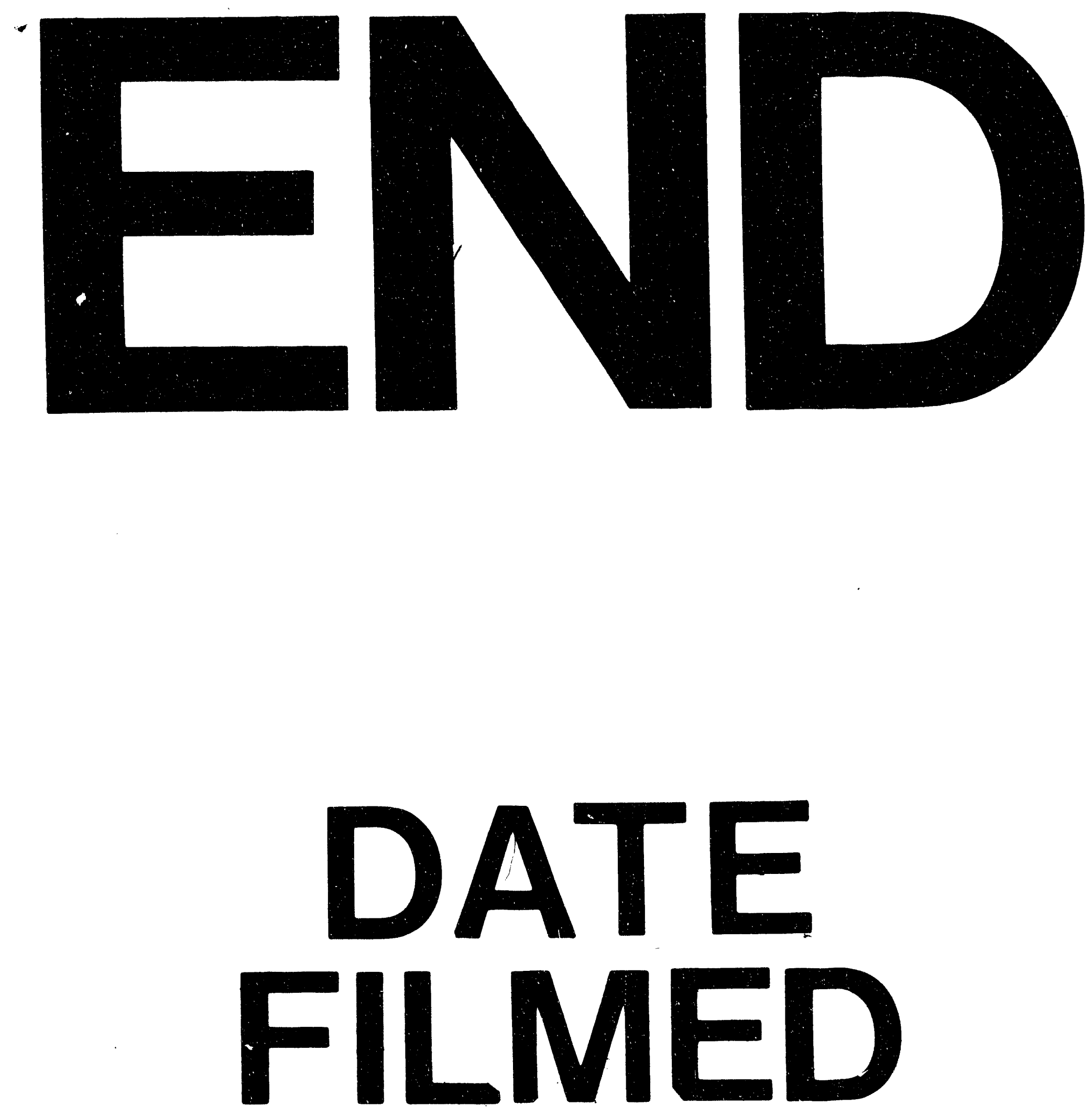

1

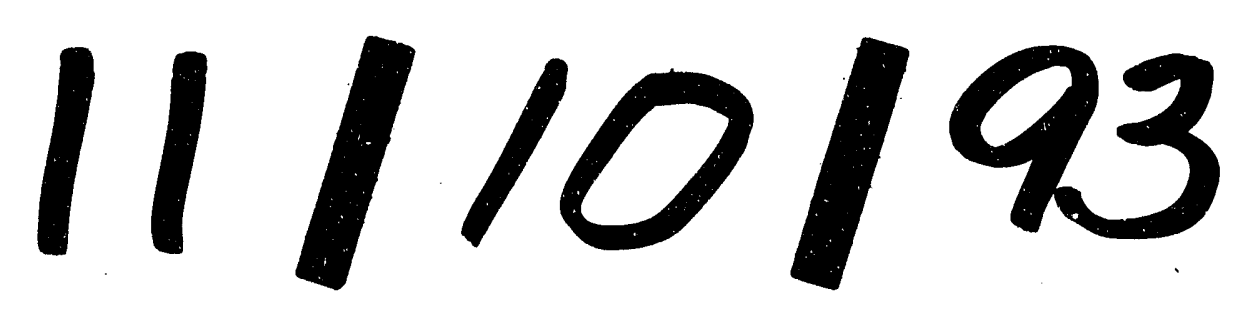


